\title{
Lymphovenous anastomosis and debulking procedure for treatment of combined severe lower extremity and genital lymphedema: A case report
}

\author{
Mario F. Scaglioni MD* (i) | Semra Uyulmaz MD2*
}

${ }^{1}$ Department of Hand and Plastic Surgery, Luzerner Kantonsspital, Lucerne, Switzerland

${ }^{2}$ Department of Plastic and Hand Surgery, University Hospital Zurich, Zurich,

Switzerland

\section{Correspondence}

Mario F. Scaglioni, MD, Department of Hand and Plastic Surgery, Luzerner Kantonsspital, Lucerne, Switzerland. Email: Mario.scaglioni@gmail.com

\begin{abstract}
Lymphedema most commonly occurs after cancer treatment and can affect limbs and genitalia. Genital lymphedema (GL) is a rare condition and can be disabling psychologically and physically. It often occurs along with lower extremity lymphedema (LEL). Conservative and physiologic reconstructive surgery such as lymphaticovenous anastomosis (LVA) offer good treatment options for LEL. GL however remains a reconstructive dilemma. The most effective surgical therapies in advanced GL are still debulking procedures in properly selected patients. Here, we present the surgical treatment of a 51 -year-old male patient with advanced and combined genital and right lower extremity lymphedema after Hodgkin lymphom treatment in the childhood. We performed multiple LVA to the right ankle joint, distal lower leg and lateral knee and 2 months later patient reported a significant decrease of pain and pressure in affected limb while the scrotal and penis lymphedema did not show any signs of improvement at all. Four months later, $4.9 \mathrm{~kg}$ of excessive lymphedematous tissue from the genital area was resected and covered by split-thickness skin grafts from the unaffected left upper thigh. The postoperative course was uneventful and 3 weeks postoperatively the skin graft healed completely. Follow up at 6 months showed reasonable cosmetic and functional outcomes and the patient reported a significant improvement of quality of life. We believe that debulking procedures and LVA may be combined in advanced GL and LEL and may provide good outcomes.
\end{abstract}

\section{1 | INTRODUCTION}

Lymphedema is a chronic condition of localized protein rich interstitial fluid retention and tissue remodeling caused by a compromised lymphatic system. The condition can be disabling psychologically and physically. Symptoms include swelling, recurrent skin infections and impairment of functionality. In developed countries lymphedema most commonly occurs after radical cancer treatment and mainly affects limbs. Genital lymphedema (GL) is a rare condition but often occurs along with lower extremity lymphedema (LEL). While complex physical therapy (CPT) and physiologic reconstructive surgery such as lymphaticovenous anastomosis (LVA) offer good treatment options for LEL, GL remains a challenging disease due to the anatomical and physiological characteristic of the genital region and a major excision procedure is often necessary (Chang, Suami, \& Skoracki, 2013; Singh, Sinha,

*Mario F. Scaglioni and Semra Uyulmaz contributed equally to this work.
Sankhwar, \& Kumar, 2011). The most effective surgical therapies in advanced GL are still debulking procedures and when applied in properly selected patients, functional and cosmetic results are promising (McDougal, 2003).

Here, we report the case of a patient affected by an advanced LEL and GL. The surgical treatments included LVA and debulking procedure and functional and aesthetic outcomes are demonstrated.

\section{I CASE REPORT}

A 51-year-old male patient was referred to our department for surgical lymphedema treatment in 2017. Patient had suffered from Hodgkin's lymphoma as a 7-year-old child and underwent inguinal lymphadenectomy and radiotherapy of the groin region in 1973. Right lower limb and genital lymphedema became clinically manifest in 1988. Since childhood, the patient was under long-term CPT that involved meticulous skin care, lymphatic drainage, compression bandaging and later 


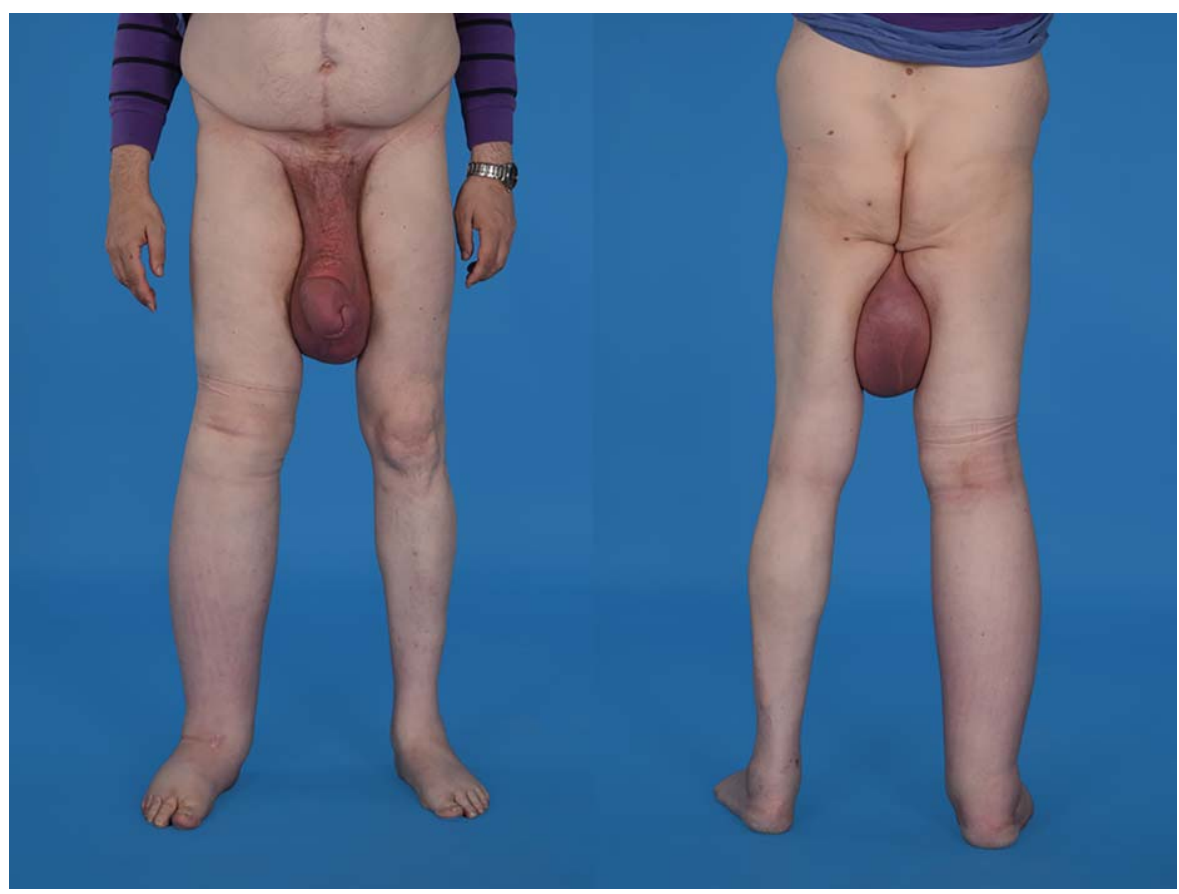

FIGURE 1 Preoperative pictures of the lymphedema involving right lower leg and genital region

garments of the right lower extremity up to the scrotum. Vascular ultrasonography was performed to rule out any venous anomaly. The excessive scrotal skin was thickened and very fragile. The penis was almost completely buried under the excessive pubic and scrotal tissue. In the groin and scrotal region lymphorrhea was present and resistant to former laser coagulation therapies. The right lower limb was swollen, the skin was thickened and positive Stemmer's sign of the dorsal foot yielded the clinical diagnosis. Lymphoscintigraphy and mainly indocyanine green (ICG) lymphography with multiple site injections were performed for supporting diagnosis and further classification of the lymphedema.

At the age of 51, the patient presented with a secondary International Society of Lymphology (ISL) stage II lymphedema of the right lower extremity and ISL stage III lymphedema of the scrotum and penis (Figure 1). Recurrent skin infections, lymphorrhea, and chronic pain complicated the entire history of the patient who was socially isolated and sexually limited due to this condition.

We performed four LVA supermicrosurgeries at the level of the right ankle joint, distal lower leg, and lateral knee; an immediate softening of the dorsal foot was noticed intraoperatively (Figure 2). Afterward, the scrotal basis was explored bilaterally with a small incision and ICG examination was performed intraoperatively. Only sclerotic and fibrotic lymphatics were detected clinically with no signs of lymphatic drainage. LVA was not seen as a possible option in this region. The postoperative course was uneventful and the patient continued wearing compression garments. At 3-weeks postoperative the circumference of the affected limb had not changed significantly due to long lymphedema history, while the edema had become softer and more bearable for the patient. Patient reported a significant decrease of pressure sensation in affected limb. The scrotal lymphedema though did not show any signs of improvement at all. Four months later a debulking procedure of the excessive tissue of the scrotum and penis was planned. A total of $4.9 \mathrm{~kg}$ tissue was removed. The testicles were preserved and relocated into a surgically prepared subcutaneous pocket in the inguinal regions on both sides. The resected areas including the whole penile shaft were covered with split-thickness skin grafts from patient's unaffected upper thigh (Figure 3). The post-operative course was uneventful and 3 weeks postoperatively the skin graft healed completely. Patient reported a significant change in quality of life and penile erection was possible. Follow up at 6 months showed reasonable cosmetic and functional outcomes. (Figure 4).

\section{3 | DISCUSSION}

Damage in the lymphatic system manifests itself as a lymphedema. Radical lymphadenectomy and radiotherapy, which are strongly associated with the development of a lymphedema, are still essential components of the therapeutic concept of cancer. The incidence of lymphedema in survivors of cancer in general is reported in the literature as 15\% (Cormier et al., 2010). LVA has been shown to be highly effective in limb lymphedema (Chang et al., 2013; Koshima, Inagawa, Urushibara, \& Moriguchi, 2000). GL in general is a rare entity and remains a reconstructive dilemma. However, it is a common complication after radical pelvic or inguinal lymphadenectomy and radiotherapy and usually manifests itself along with LEL. Patients usually tend to conceal the disease due to embarrassment, which results in late diagnosis and treatment. Swelling, lymphorrhea, fatigue, pain, and recurrent subcutaneous infections due to the difficulty of self-hygiene may be present in GL patients. Eventually aesthetic and functional disabilities impair patients' quality of life immensely (Singh et al., 2011). GL treatment is challenging and not yet established. The anatomical complexity of the genital regions makes it difficult for physiotherapists for CPT. 

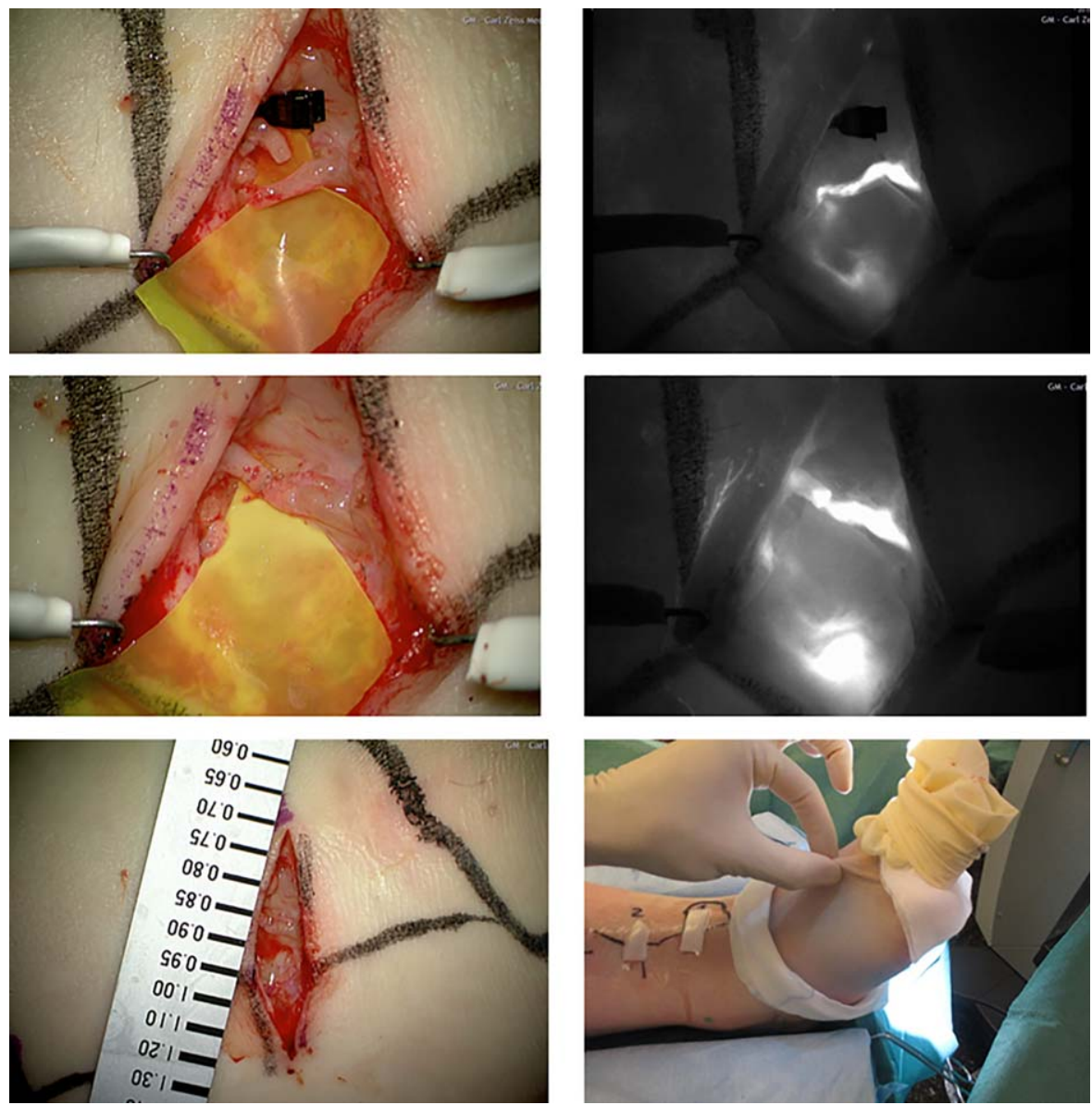

FIGURE 2 Dissection of a Lymphatic duct at the ankle level (above left), demonstration of the lymphatic duct with ICG (above right), LVA (middle left), LVA with ICG (middle right), the caliber of the LVA was measured: $0.85 \mathrm{~mm}$ (below left), intraoperative lymphedema reduction at the right dorsal foot just after the LVA operation (below right)
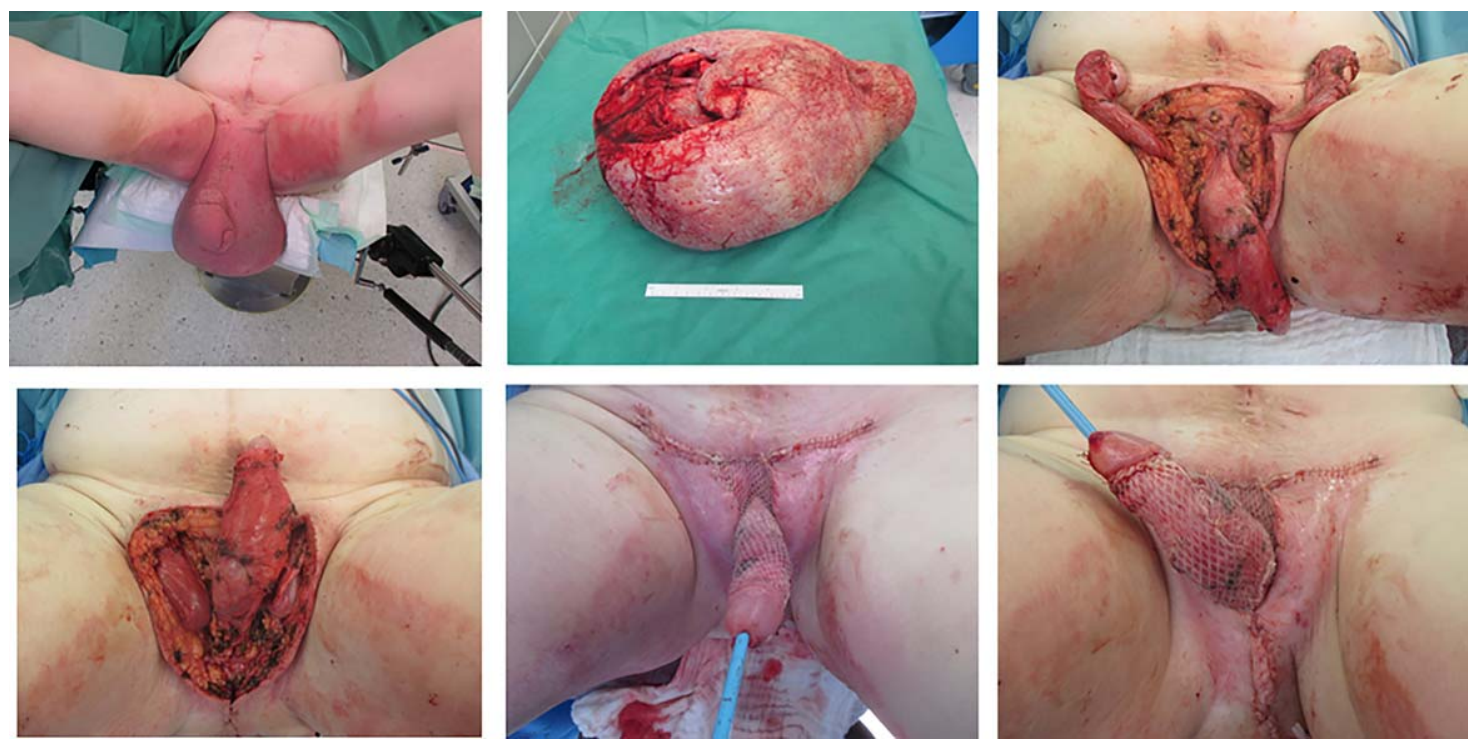

FIGURE 3 Genital lymphedema (above left), specimen of the resected lymphedematous tissue (above middle), penis and testis after the resection (above left), the testis were relocated in a subcutaneous pocket in the inguinal region bilaterally (below right), penile shaft was covered with STSG (below middle and right) 


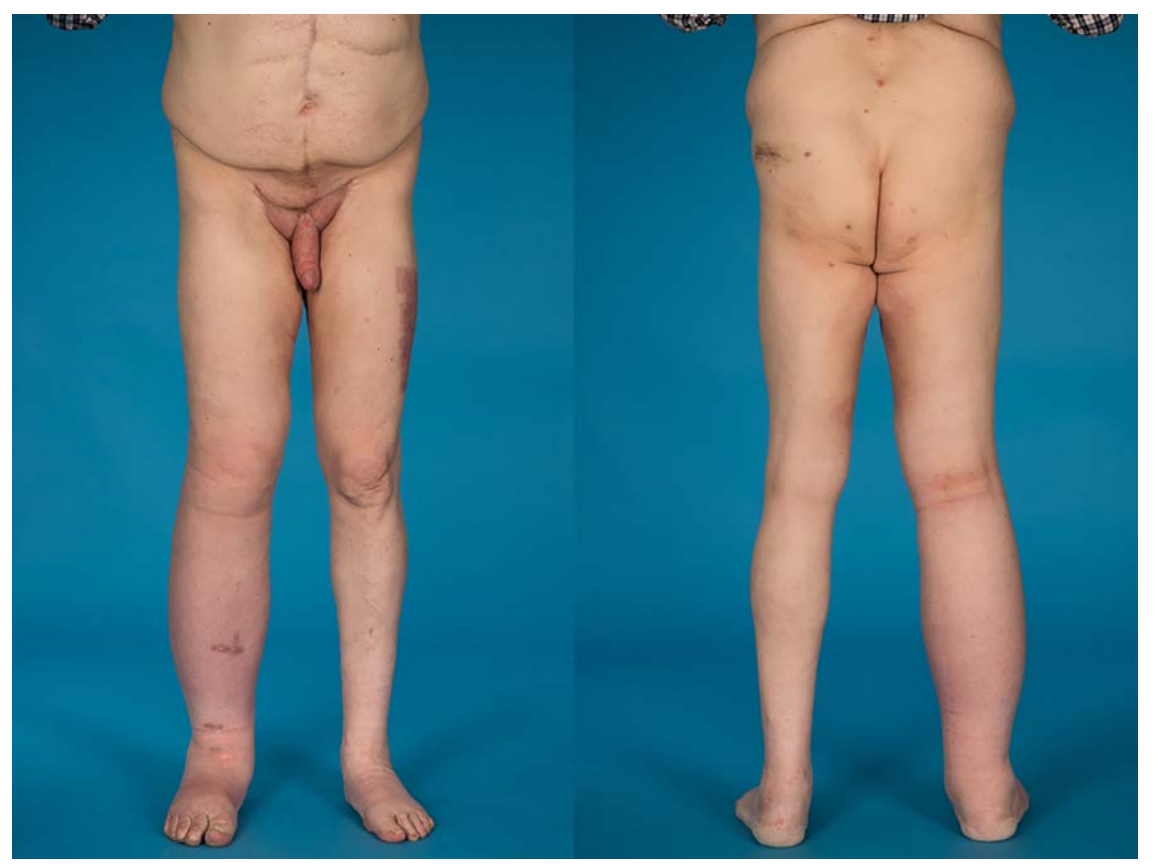

FIGURE 4 Postoperative pictures at 6 months after LVA and debulking procedure for treatment of combined severe lower extremity and genital lymphedema

Lymphatic flow around the genital region is difficult to understand, especially in lower limb lymphedema patients (Hara \& Mihara, 2017). ICG lymphography allows visualization of abnormal lymph circulation in the lower abdominal and the genital region, which plays an important role in early diagnosis and treatment of GL (Yamamoto et al., 2013). It was shown to be a more sensitive method to examine lymphatic pathways of the genital regions than lymphoscintigraphy. We applied additional multisite ICG injections in the lower abdomen, scrotum and lower leg preoperatively and examined lymph circulation in the genital area with a delay of at least $30 \mathrm{~min}$.

Surgical treatment is indicated in cases of moderate to severe genital lymphedema (Parmar, 2013). Clodius, et al. discussed the ineffectiveness of microsurgery on altered tissue due to elephantiasis in 1981. However, treatment of GL with LVA was described in more recent literature (Clodius, Piller, \& Casley-Smith, 1981). Mukenge et al. 2007 reported a case of secondary LEL and GL, which was successfully treated performing LVA on the genital region and lower extremities (Mukenge, Pulitanò, Colombo, Negrini, \& Ferla, 2007). Yamamoto et al. (2011) performed simultaneous multisite LVA in patients with GL accompanying lymphorrhea with excellent results (Yamamoto et al., 2011). However restoring the lymphatic drainage with LVA is limited to cases with minor stasis, well-isolated lymphatic channels and no fibrosis (Mukenge et al., 2011). Thus, the optimal operative technique and timing of the genital debulking procedures remain controversial. Most commonly the most effective surgical therapy in advanced GL remains excision and, when applied in properly selected patients, functional and cosmetic results are promising (McDougal, 2003). Debulking techniques for the management of this condition can involve partial or total resection of the skin and the subcutaneous tissue associated with lymphatic drainage. In 1912 the Charles procedure was introduced as a debulking procedure when surgeons excised excessive subcutaneous and deep fascial tissue in lower limb lymphedema circumferentially and resurfaced the extremity with split-thickness grafts taken from the excised tissue (Charles, 1912). Exophytic changes within the grafted skin, hypertrophic scarring, chronic cellulitis and skin breakdown led to deformities that were significantly worse than the original limb lymphedema. Indeed, the concept of split skin grafting is considered as outdated and whenever possible, regional skin flap should be used preferably (Kumar \& Manokaran, 2015). In our case, the patient had previous irradiation of the inguinal and lower abdominal region and the skin quality was very poor. The penile foreskin was indeed affected by lymphedema. Moreover, our patient rejected any local flap coverage from the thigh. However, application of a split-thickness skin graft involving the penile shaft had been shown to promote adequate skin coverage, with a penile girth compatible with sexual intercourse and little alteration in sensitiveness by Modolin et al. in 2006 (Modolin et al., 2006). It can be hypothesized that ablative surgery only cosmetically and temporarily improves the lesion and that it most likely will result in disease recurrence because it does not resolve the physiological cause of GL. Nonetheless, contour resection does not only obviously decrease bulk but also improve appearances. Several studies have shown that debulking procedures in patients with genital lymphedema lead to a significant increase in quality of life and to a significant reduction of skin infection rates (Singh et al., 2011; Zvonik, Földi, \& Felmerer, 2011). When combined with lymphaticovenous anastomosis alongside affected limbs, the lymphatic drainage can be improved and the overall outcome might even be increased.

To our knowledge, even if these surgical procedures are not novel, this two-staged approach along with multiple LVA for combined limb and genital lymphedema has not been reported in literature so far. We 
believe that surgical resection of excessive tissue in advanced GL may be combined in exceptional situations with lymphaticovenous anastomosis of the lower extremity to provide better outcomes.

\section{DISCLOSURE}

The authors have no financial conflicts or commercial associations to disclose.

\section{ORCID}

Mario F. Scaglioni MD (iD http://orcid.org/0000-0001-5289-9140

\section{REFERENCES}

Chang, D. W., Suami, H., \& Skoracki, R. (2013). A prospective analysis of 100 consecutive lymphovenous bypass cases for treatment of extremity lymphedema. Plastic \& Reconstruction Surgery, 132, 13051314.

Charles, H. (1912). Elephantiasis of the leg. In A. Latham \& T. C. English (Eds.), A system of treatment (Vol. 3). London: Churchill.

Clodius, L., Piller, N. B., \& Casley-Smith, J. R. (1981). The problems of lymphatic microsurgery for lymphedema. Lymphology, 14, 69-76.

Cormier, J. N., Askew, R. L., Mungovan, K. S., Xing, Y., Ross, M. I., \& Armer, J. M. (2010). Lymphedema beyond breast cancer: A systematic review and meta-analysis of cancer-related secondary lymphedema. Cancer, 116, 5138-5149.

Hara, H., \& Mihara, M. (2017). Indocyanine green lymphographic and lymphoscintigraphic findings in genital lymphedema-Genital pathway score. Lymphatic Research and Biology, 15, 356-359.

Koshima, I., Inagawa, K., Urushibara, K., \& Moriguchi, T. (2000). Supermicorsurgical lymphaticovenular anastomosis for the treatment of lymphedema in the upper extremities. Journal of Reconstructive Microsurgery, 16, 437-442.

Kumar, K. L. \& Manokaran, G. (2015). Genital lymphedema: A new technique of correction surgery. Lymphology, 48, 153-156.

McDougal, W. S. (2003). Lymphedema of the external genitalia. Journal of Urology, 170, 711-716.
Modolin, M., Mitre, A. I., da Silva, J. C., Cintra, W., Quagliano, A. P. Arap, S., \& Ferreira, M. C. (2006). Surgical treatment of lymphedema of the penis and scrotum. Clinics (Sao Paulo), 61, 289-294.

Mukenge, S. M., Catena, M., Negrini, D., Ratti, F., Moriondo, A., Briganti, A., Rigatti, P., Cipriani, F., \& Ferla, G. (2011). Assessment and followup of patency after lymphovenous microsurgery for treatment of secondary lymphedema in external male genital organs. European Urology, 60, 1114-1119.

Mukenge, S., Pulitanò, C., Colombo, R., Negrini, D., \& Ferla, G. (2007). Secondary scrotal lymphoedema: A novel microsurgical approach. Microsurgery, 27, 655-656.

Parmar, H. D. (2013). The surgical approach in huge scrotal lymphedema. International Journal of Medical Science \& Public Health, 2, 153-155.

Singh, V., Sinha, R. J., Sankhwar, S. N., \& Kumar, V. (2011). Reconstructive surgery for penoscrotal filarial lymphedema: A decade of experience and follow-up. Urology, 77, 1228-1231.

Yamamoto, T., Koshima, I., Yoshimatsu, H., Narushima, M., Miahara, M., \& lida, T. (2011). Simultaneous multi-site lymphaticovenular anastomoses for primary lower extremity and genital lymphoedema complicated with severe lymphorrhea. Journal of Plastic, Reconstructive \& Aesthetic Surgery, 64, 812-815.

Yamamoto, T., Yamamoto, N., Yoshimatsu, H., Hayami, S., Narushima, M., \& Koshima, I. (2013). Indocyanine green lymphography for evaluation of genital lymphedema in secondary lower extremity lymphedema patients. Journal of Vascular Surgery: Venous \& Lymphatic Disorders, 1, $400-405$

Zvonik, M., Földi, E., \& Felmerer, G. (2011). The effects of reduction operation with genital lymphedema on the frequency of erysipelas and the quality of life. Lymphology, 44, 121-130.

How to cite this article: Scaglioni MF, Uyulmaz S. Lymphovenous anastomosis and debulking procedure for treatment of combined severe lower extremity and genital lymphedema: A case report. Microsurgery. 2018;38: 907-911. https://doi.org/ 10.1002/micr.30331 